
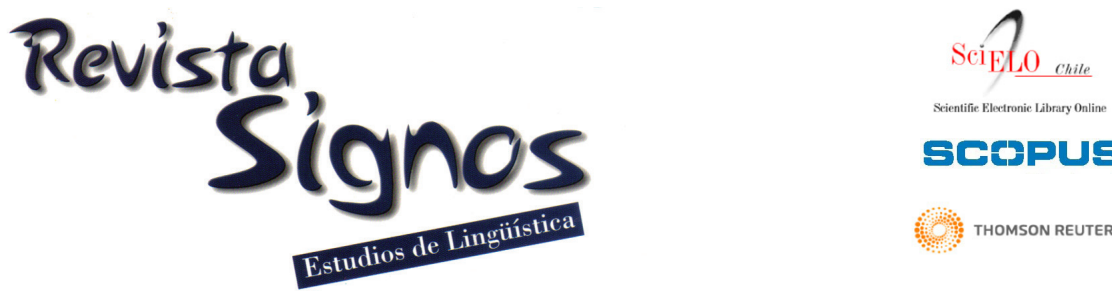

\title{
Aproximación empírica a los modos de acción del verbo: Un estudio basado en corpus*
}

\author{
An empirical approach to Aktionsart: A corpus-based study
}

\author{
Marta Coll-Florit \\ mcollfl@uoc.edu \\ Universitat Oberta de Catalunya \\ España
}

Recibido: I0-VIII-20I0 / Aceptado: I0-V-20II

\begin{abstract}
Resumen: En este artículo se presenta un estudio sobre los modos de acción del verbo basado en datos del español extraídos de corpus. El trabajo incluye una muestra amplia y variada de predicados verbales, así como de construcciones gramaticales, con el propósito fundamental de aportar datos empíricos sobre uno de los temas más prolíficos de las aproximaciones teóricas a la semántica léxica. En particular, en el estudio se abordan dos objetivos básicos: por un lado, identificar las diferentes restricciones de coaparición 'verbo + construcción gramatical' en función de la categoría aspectual del verbo; y por otro lado, describir cómo se estructuran internamente dichas categorías. Los resultados muestran claramente que las diferentes categorías de los modos de acción del verbo usan diferentes patrones morfosintácticos.Además, se demuestra que dichas categorías, lejos de estructurarse a partir de condiciones necesarias y suficientes, presentan una estructura interna gradual, con verbos prototípicos que no admiten cambios de interpretación aspectual y verbos más flexibles que se pueden adaptar a otras categorías.
\end{abstract}

Palabras Clave: Semántica verbal, modos de acción del verbo, lingüística de corpus, prototipicidad. 


\begin{abstract}
This paper presents a comprehensive study on Aktionsart based on Spanish data extracted from corpora. This work includes a large and diverse set of verbal predicates as well as grammatical constructions, with the primary purpose of providing empirical evidence on one of the most prolific topics in the theoretical approaches to lexical semantics. In particular, the main goal of this paper is twofold: firstly, to identify different restrictions in the interaction of 'verb + grammatical construction' depending on the aspectual category of the verb; and secondly, to describe how these categories are internally structured. The results clearly show that the different Aktionsart categories use different morphosyntactic patterns. Moreover, it is demonstrated that these categories, far from being structured from necessary and sufficient conditions, present a gradual internal structure, with prototypical verbs which do not accept aspectual shifting and more flexible verbs which can be adapted to other categories.
\end{abstract}

Key Words:Verbal semantics, Aktionsart, corpus linguistics, prototypicality.

\section{INTRODUCCIÓN}

Desde la lingüística teórica se considera que una propiedad fundamental de la semántica verbal es el modo de acción (también denominado 'aspecto léxico', 'Aktionsart' o 'tipo de situación'), esto es, la información relacionada con la estructura temporal interna del evento descrito por un predicado verbal: si es estático o dinámico; si se extiende a lo largo de un periodo temporal o es puntual; si implica una culminación inherente o está exento de delimitación; entre otras distinciones. A partir de la combinación de estas oposiciones nocionales básicas se han propuesto tipologías de clasificación verbal que se consideran útiles para predecir el comportamiento sintáctico de los predicados. En concreto, la propuesta más influyente de los últimos 50 años es la de Vendler (1957), que distingue cuatro clases de predicados verbales: estados, actividades, realizaciones y logros.

No obstante, lejos de ser un ámbito de estudio con unos principios teóricos y metodológicos consensuados, la bibliografía sobre los modos de acción del verbo se caracteriza por la multiplicidad de propuestas de clasificación, que no solo difieren en cuanto al número y organización de las clases aspectuales, sino también en el nivel lingüístico en el cual se manifiestan dichas clases (Dowty, 1979; Verkuyl, 1989; Mittwoch, 199I; Pustejovsky, 1991; Smith, I991; Levin \& Rappaport Hovav, 1995; De Miguel, 1999, 2004; Marín, 2000; Croft, 2008; entre otros). En este sentido, y de manera más relevante, no hay acuerdo a la hora de abordar el problema teórico de cómo y en qué medida interactúan la semántica léxica y la sintaxis en la codificación lingüística de la información aspectual. Respecto a la metodología, es difícil encontrar estudios empíricos que contengan un número significativo de predicados verbales: generalmente los trabajos sobre los modos de acción del verbo se basan en análisis cualitativos de un conjunto reducido de predicados. De este modo, observamos que las divergencias teóricas, así como las carencias metodológicas, ponen de manifiesto la necesidad de estudios más exhaustivos y empíricos sobre la materia.

Este trabajo pretende dar respuesta a dichas necesidades. En particular, se presenta un estudio de corpus, basado en una muestra amplia y variada de verbos del español, con el fin de obtener evidencia empírica de su comportamiento morfosintáctico en contextos de uso reales. Más concretamente, los objetivos básicos del estudio son dos: por un lado, verificar que se dan diferentes restricciones de coaparición 'verbo + construcción gramatical' en función de la categoría aspectual del verbo; y por otro lado, describir el modo de estructuración interna de dichas categorías. Así, el presente estudio nos permitirá obtener una clasificación de los modos de acción del verbo probada empíricamente $y$, de manera relacionada, una nueva metodología para la identificación de rasgos semánticos.

\section{Marco teórico}

Uno de los debates más prolíficos en la bibliografía sobre los modos de acción del verbo se refiere al nivel lingüístico de gramaticalización de los eventos: ¿es una propiedad de la pieza léxica verbal o de toda la oración? Siguiendo a De Miguel (2004) y Demonte (2006), se pueden distinguir dos grandes aproximaciones: por un lado, los modelos lexicalistas (Jackendoff, 1990, 2002; Levin \& Rappaport Hovav, 1995) asumen que las propiedades semánticas listadas en el lexicón verbal -incluidas las distinciones aspectuales- proyectan, y por lo tanto determinan, la estructura sintáctica del verbo; contrariamente, los 
modelos neoconstruccionistas (Borer, 1994; Ritter \& Rosen, 1998) consideran que el aspecto es una propiedad sintáctica y que la semántica léxica tiene una influencia muy limitada en el comportamiento sintáctico del verbo.

En este trabajo, en la línea de los modelos lexicalistas, partimos de la hipótesis básica de que el verbo presenta propiedades semánticas relacionadas con el aspecto que imponen ciertas restricciones de coaparición contextual.Así, si observamos el ejemplo (I), vemos que el verbo 'perseguir', que léxicamente denota un proceso atélico, no puede coaparecer con el sintagma adverbial delimitador 'en dos minutos'.

(I) * La policía persiguió al ladrón en dos minutos.

Asimismo, entendemos que el modo de acción es composicional (Verkuyl, 1989; Marín, 2000). Es decir, asumimos que el verbo denota un significado aspectual básico que puede ser modificado a nivel oracional, en función de las propiedades cuantificacionales de sus argumentos, del tiempo verbal en que se conjuga el verbo o de los adjuntos que lo complementan, entre otras posibles modificaciones. No obstante, a diferencia de la visión composicional tradicional, partimos de la hipótesis básica de que se establecen diferentes pesos léxicos o grados de prototipicidad en la interacción entre verbo y contexto oracional. Por ejemplo, en las oraciones de (2) aparecen dos verbos que típicamente se han considerado estados, 'constar' y 'conocer'. Aun así, vemos que el verbo 'conocer' es mucho más flexible que el verbo 'constar' al aparecer en contextos dinámicos, como es el caso del modificador adverbial 'poco a poco' o la perífrasis progresiva.

(2) a. * Su nombre poco a poco está constando de cinco letras.

b. Silvia poco a poco está conociendo a Pedro.

Por lo tanto, entendemos que las categorías aspectuales presentan miembros más prototípicos y miembros más fronterizos o flexibles. En particular, dependiendo del grado de flexibilidad aspectual que admite un verbo, asumimos que se pueden identificar tres grandes tipos de predicados: verbos monosémicos estables, verbos monosémicos flexibles y verbos polisémicos, aspectualmente hablando. Una tipología que se refleja en diferentes grados de estabilidad semántica, sintáctica y morfológica.
Más concretamente, los verbos monosémicos estables (ej. 'constar') son los que presentan un único tipo básico de papeles temáticos, imponen fuertes restricciones de realización sintáctica y muestran una preferencia por un determinado tiempo verbal. Estos verbos no admiten cambios de interpretación aspectual, por lo que son miembros prototípicos de una categoría aspectual. En una posición intermedia encontramos los verbos monosémicos flexibles (ej. 'conocer') que también presentan un único tipo básico de papeles temáticos; no obstante, a diferencia de los verbos monosémicos estables, imponen restricciones morfosintácticas más débiles, por lo que admiten cambios de interpretación aspectual a partir de la composición. Finalmente, en el otro extremo, se sitúan los verbos aspectualmente polisémicos (ej. 'contener' [+ materia] vs 'contener' [+ emociones]; 'salir de/ a' [+ lugar] vs 'salir a' [+ cantidad]) que presentan como mínimo dos sentidos léxicos que pertenecen a diferentes categorías aspectuales -de manera estable o flexible-. Cada sentido requiere diferentes papeles temáticos e impone diferentes restricciones morfosintácticas. Observamos que, según este enfoque, los verbos pueden imponer diferente peso léxico en la gramaticalización de la información aspectual. En consecuencia, nuestra propuesta teórica se configura como una posición intermedia entre los modelos lexicalistas y los modelos neoconstruccionistas.

El presente trabajo se centra en el estudio de los verbos que hemos denominado aspectualmente monosémicos (estables y flexibles). Más concretamente, las hipótesis de partida se pueden sintetizar de la siguiente manera:

El verbo incorpora propiedades semánticas relacionadas con el aspecto que imponen restricciones contextuales. En consecuencia, se espera que las categorías aspectuales léxicas, comparadas entre sí, presenten diferentes patrones generales de uso.

Las categorías aspectuales no se estructuran a partir de condiciones necesarias y suficientes. Por el contrario, presentan una organización interna gradual, con miembros prototípicos que no admiten cambios de interpretación aspectual y miembros más flexibles que, a través de los principios de la composición, se pueden mover hacia otras categorías aspectuales. 


\section{Metodología}

Con el fin de probar empíricamente estas hipótesis, realizamos un estudio de corpus. En particular, nos basamos en un subcorpus de 81 millones de palabras del Corpus de Referencia del Español Actual (CREA) de la Real Academia Española (http:// corpus.rae.es/creanet.html), que incluye textos de los últimos 25 años. La elección del corpus se fundamenta en dos criterios básicos: la extensión y variedad de textos, así como el prestigio del que goza en el mundo académico.

En cuanto a la muestra verbal, se analizaron un total de 120 predicados verbales del español, 30 predicados para cada una de las cuatro categorías básicas de los modos de acción del verbo:

- estados: eventos no dinámicos y durativos (ej. 'equivaler','conocer')

- procesos: eventos dinámicos, durativos y no delimitados (ej. 'caminar', 'empujar')

- realizaciones: eventos dinámicos, durativos y delimitados (ej. 'construir', 'instalar')

- logros:eventos dinámicos, puntuales y delimitados (ej.'detectar','atrapar').

En todos los casos son ítems que se han citado en la bibliografía sobre los modos de acción del verbo como ejemplos de cada una de las categorías objeto de estudio (Vendler, 1957; Dowty, 1979; Binnick, 199I; Mittwoch, I99I; Pustejovsky, 199I; Smith, 199I; Levin \& Rappaport Hovav, 1995; Havu, 1997; De Miguel, 1999, 2004; Marín, 2000; Gennari \& Poeppl, 2003; Croft, 2008; entre otros). En el Anexo se puede consultar la lista total de verbos considerados. En cuanto al sistema de análisis de la muestra verbal en el corpus, nos basamos en lexemas con el fin de obtener datos de todas las formas verbales de los ítems analizados. Este sistema es especialmente relevante para poder dar cuenta de los cambios de interpretación del modo de acción del verbo provocados por la flexión de tiempo y aspecto (ver sección 3.5).

En relación a la muestra de contextos, se analizaron 14 de las construcciones gramaticales que en la bibliografía se usan de forma más recurrente para identificar los parámetros de la dinamicidad, la delimitación y la duración -parámetros que sustentan la tipología aspectual cuatripartita de la que parte el presente estudio-, y se aplicaron a todos los verbos de la muestra. De este modo, se obtuvieron un total de 1.680 contextos [ 120 verbos x 14 construcciones]. La Tabla I presenta la lista completa de las construcciones gramaticales analizadas.

Tabla I. Muestra de construcciones gramaticales.

\begin{tabular}{|c|c|}
\hline \multicolumn{2}{|c|}{ I. Perífrasis progresiva } \\
\hline \multicolumn{2}{|c|}{ 2.Adverbio 'lentamente’ } \\
\hline \multicolumn{2}{|c|}{ 3.Adverbio 'rápidamente' } \\
\hline \multicolumn{2}{|c|}{ 4. Interpretación habitual: <cada díal semanal mes/ año $+V_{\text {presente }}>$} \\
\hline \multicolumn{2}{|c|}{ 5. <obligar $a+V_{\text {infinitivo }}>$} \\
\hline \multicolumn{2}{|c|}{ 6. <durante $X$ tiempo> } \\
\hline \multicolumn{2}{|c|}{ 7. <en X tiempo> } \\
\hline \multicolumn{2}{|c|}{ 8. $<$ acabar de $+V_{\text {infinitivo }}>$} \\
\hline \multicolumn{2}{|c|}{ 9. <de repente> } \\
\hline \multicolumn{2}{|c|}{ 10. <a las $X$ horas $>$} \\
\hline \multicolumn{2}{|c|}{ II. <desde hace $X$ tiempo> } \\
\hline \multirow[t]{3}{*}{ Tiempo verbal } & 12. Presente \\
\hline & I3. Imperfecto \\
\hline & 14. Pasado simple \\
\hline
\end{tabular}


En cuanto al procedimiento de análisis de los datos, se dividió en dos fases:

a) Análisis intercategorial. El primer objetivo era comprobar si las cuatro categorías básicas de Aktionsart, comparadas entre sí, mostraban diferentes patrones de uso morfosintáctico. A tal fin, se analizaron cada una de las construcciones gramaticales por separado para el conjunto total de categorías aspectuales (estados, procesos, realizaciones y logros). Con este procedimiento pudimos identificar cuál es la categoría que aparece con más frecuencia con cada construcción, así como identificar la categoría que presenta los índices de frecuencia más bajos.

b) Análisis intracategorial. El segundo objetivo era analizar la estructura interna de las categorías aspectuales, con el fin de comprobar si presentan diferentes grados de prototipicidad. En consecuencia, se analizaron cada una de las categorías por separado para el conjunto total de construcciones gramaticales. De este modo, pudimos identificar cuál es el conjunto de verbos de una categoría que presenta los índices más altos y más bajos de frecuencia para cada construcción, así como detectar los rasgos semánticos compartidos por los diferentes subgrupos verbales.

Esta metodología pretende reflejar la gradación de las categorías aspectuales a dos niveles, en la línea de la distinción postulada por Aarts (2004) entre Subsective Gradience (SG) y Intersective Gradience (IG): por un lado, la SG, inherentemente intracategorial, permite que los miembros de una categoría manifiesten las propiedades de dicha categoría a diferentes niveles; por otro lado, la IG, inherentemente intercategorial, se refiere a la intersección de un conjunto de propiedades entre categorías, sin implicar una intersección total de las categorías. De este modo, la presente concepción de la gradación se sitúa en una posición intermedia entre los modelos que parten de una visión aristotélica de la categorización y los modelos que postulan una gradación sin restricciones, esto es, sin transiciones claras entre categorías.
Dedicamos los siguientes apartados a presentar los principales resultados de ambos análisis: 'intercategorial' (sección 3) e 'intracategorial' (sección 4).

\section{Resultados del análisis intercategorial}

El presente apartado reseña los principales resultados del análisis 'intercategorial'. En particular, se analizan los diferentes patrones de uso mofosintáctico de cada una de las cuatro categorías básicas del modo de acción del verbo, para cada una de las construcciones gramaticales de la muestra.

\section{I. Perífrasis progresiva: <estar $+V_{\text {gerundio }}>$}

Desde el trabajo de Vendler (1957) se ha tendido a asumir que la forma progresiva (<estar $+\mathrm{V}_{\text {gerundio }}>$ ), al implicar sucesión de diversas fases temporales, solo es aceptada por verbos que expresan eventos dinámicos durativos. No obstante, si bien en ciertas lenguas como el francés, el italiano o el finés la incompatibilidad de los estados con la forma progresiva se da de forma sistemática (Bertinetto, 1987; Havu, 1997), en otras lenguas como el español no siempre se mantiene (De Miguel, 1999). En esta línea, varios autores reivindican el hecho de que muchos logros admiten esta construcción (Mourelatos, I978; Verkuyl, I989).

Según los resultados del presente estudio de corpus, hemos detectado que todas las categorías aspectuales aceptan, en algún caso, la perífrasis progresiva. Sin embargo, se dan claras diferencias de frecuencia de coaparición entre categorías. En concreto, los datos muestran que los verbos que expresan eventos dinámicos durativos y no delimitados (procesos) son los que más aceptan la forma progresiva, seguidos de los verbos que expresan eventos dinámicos durativos y delimitados (realizaciones). Finalmente, los verbos que designan eventos dinámicos puntuales (logros) y los que expresan eventos no dinámicos durativos (estados) son los más reticentes a aparecer con esta construcción. En la Tabla 2 se presentan los porcentajes totales de frecuencia para

Tabla 2. Perífrasis progresiva.

\begin{tabular}{|c|c|c|c|}
\hline Procesos & Realizaciones & Logros & Estados \\
\hline $58,54 \%$ & $26,83 \%$ & $9,76 \%$ & $4,88 \%$ \\
\hline
\end{tabular}


cada categoría, de más a menos frecuentes, con una disposición de izquierda a derecha.

\section{2. 'Lentamente'y 'rápidamente'}

Otro de los criterios más utilizados para identificar la progresión temporal del evento expresado por un verbo es el de los llamados adverbios de temporalidad interna, como 'lentamente' y 'rápidamente' (De Miguel, 1999; Marín, 2000). Por lo tanto, se espera una distribución intercategorial equivalente a la de la perífrasis progresiva. Los resultados verifican esta predicción para 'lentamente' (ver Tabla 3): los procesos presentan los índices de coaparición más altos, seguidos de las realizaciones y los logros.

En cuanto al adverbio 'rápidamente', hay que destacar dos rasgos diferenciales con respecto a las distribuciones de la perífrasis progresiva y el adverbio 'lentamente'. Por un lado, si observamos la Tabla 4, vemos que en este caso los índices de frecuencia de las realizaciones superan los índices de los procesos. Por otro lado, no encontramos diferencias de frecuencia relevantes entre procesos y logros, categorías contiguas en esta distribución. De este modo, todos los eventos dinámicos, ya sean durativos o puntuales, muestran claras diferencias de coaparición con el adverbio 'rápidamente' en comparación con la categoría de los estados.

Sin embargo, es importante notar que, en contra de las predicciones tradicionales, encontramos algunos casos de estados con el adverbio 'rápidamente' (ej. 'Conocí rápidamente a los demás parceleros').

\subsection{Interpretación habitual: <cada díal semanal mesl año $+V_{\text {presente }}>$}

Desde los modelos teóricos del modo de acción se afirma que los procesos, a diferencia de los estados, admiten una interpretación habitual cuando se conjugan en presente (Dowty, 1979; Binnick, I99I; Marín,2000). A su vez, se asume que dichas diferencias se correlacionan con la posibilidad de aparición de cada una de estas categorías con construcciones que implican reiteración, como <cada díal semanal mes/ año $+V_{\text {presente }}>$. Los resultados del estudio de corpus confirman esta hipótesis: los procesos presentan los índices de frecuencia más altos con esta construcción habitual, mientras que los estados se sitúan en el extremo menos frecuente (ver Tabla 5).

\section{4. <obligar $a+V_{\text {infinitivo }}>$}

A nivel teórico se establece un debate entre aquellos modelos que defienden una equiparación entre dinamicidad y agentividad (Lakoff, 1970; Binnick, (99I) y aquellos modelos que niegan esta equivalencia (Jackendoff, 199I; Pustejovsky, 199I). Con el fin de contrastar empíricamente esta discusión, nos hemos centrado en el análisis de la construcción agentiva <obligar $a+V_{\text {infinitivo }}>$. Según los datos obtenidos en el presente estudio, observamos que, por un lado, los eventos dinámicos durativos (realizaciones y procesos) muestran los índices más altos de coaparición con esta construcción, mientras que los logros y los estados presentan los índices más bajos (ver Tabla 6).

Tabla 3. Adverbio 'lentamente’.

\begin{tabular}{|c|c|c|c|}
\hline Procesos & Realizaciones & Logros & Estados \\
\hline $62,5 \%$ & $31,25 \%$ & $6,25 \%$ & $0 \%$ \\
\hline
\end{tabular}

Tabla 4. Adverbio 'rápidamente'.

\begin{tabular}{|c|c|c|c|}
\hline Realizaciones & Procesos & Logros & Estados \\
\hline $66,11 \%$ & $21,74 \%$ & $15,53 \%$ & $0,62 \%$ \\
\hline
\end{tabular}

Tabla 5. <cada díal semanal mes/ año $+\mathrm{V}_{\text {presente }}>$

\begin{tabular}{|c|c|c|c|}
\hline Procesos & Realizaciones & Logros & Estados \\
\hline $43,48 \%$ & $26,07 \%$ & $21,74 \%$ & $8,70 \%$ \\
\hline
\end{tabular}


Tabla 6. <obligar $a+\mathrm{V}_{\text {infinitivo }}>$

\begin{tabular}{|c|c|c|c|}
\hline Realizaciones & Procesos & Logros & Estados \\
\hline $37,84 \%$ & $35,14 \%$ & $16,21 \%$ & $10,81 \%$ \\
\hline
\end{tabular}

Tabla 7. <Durante $x$ tiempo $>$

\begin{tabular}{|c|c|c|c|}
\hline Estados & Procesos & Realizaciones & Logros \\
\hline $35,90 \%$ & $30,77 \%$ & $30,77 \%$ & $2,56 \%$ \\
\hline
\end{tabular}

Tabla 8. <en X tiempo>

\begin{tabular}{|c|c|c|c|}
\hline Realizaciones & Procesos & Logros & Estados \\
\hline $87,34 \%$ & $8,73 \%$ & $3,06 \%$ & $0,87 \%$ \\
\hline
\end{tabular}

Por lo tanto, no todos los eventos dinámicos (realizaciones, procesos y logros) se diferencian claramente de los estados en cuanto al uso de esta construcción agentiva. En consecuencia, los resultados sugieren que no se puede sostener una equiparación total entre dinamicidad y agentividad.

\section{5. <durante $X$ tiempo>, <en $X$ tiempo>}

En cuanto a las construcciones <durante $X$ tiempo> $y<$ en $X$ tiempo>, tradicionalmente se han usado como criterios para distinguir los eventos que estan exentos de límite temporal de los que implican una culminación (Vendler, 1957; Dowty, I 979; De Miguel, 1999; Levin \& Rappaport Hovav, 1995). No obstante, en relación a <durante $X$ tiempo>, los resultados del estudio de corpus muestran que los índices son prácticamente idénticos para todos los eventos durativos (ver Tabla 7).

De esta manera, la distribución de frecuencias responde a la distinción entre eventos durativos y eventos puntuales. Estos resultados se deben al hecho de que las realizaciones, aunque son inherentemente delimitadas, admiten contextos no delimitados que permiten focalizar la duración del proceso previo a la culminación del evento: inflexiones imperfectivas (3a), perífrasis de fase continuativa (3b) y objetos directos no delimitados (3c).

(3) a. La basilílica gotica se ha estado construyendo durante más de seis siglos

b. Luis González siguió escribiendo durante un tiempo su artículo en "Semana".

c. Estuvo escribiendo poemas durante algunos años.
En cuanto a la construcción <en $\mathrm{X}$ tiempo>, generalmente se ha usado como criterio aspectual opuesto al de la construcción <durante $X$ tiempo>. Así, se espera que los verbos que expresan eventos delimitados (realizaciones y logros) presenten índices más altos de coaparición con esta construcción respecto a los verbos que denotan eventos no delimitados (procesos y estados). Los resultados confirman esta hipótesis para realizaciones y estados, ya que se sitúan claramente en la posición más alta y más baja de frecuencia, respectivamente (ver Tabla 8).

No obstante, en contra de los pronósticos teóricos tradicionales, no encontramos diferencias entre procesos y logros. Estos resultados se deben al hecho de que los logros, si bien implican una culminación inherente, se caracterizan principalmente por expresar eventos puntuales, razón por la cual solo aceptan la construcción <en $\mathrm{X}$ tiempo> de forma ocasional con medidas de tiempo muy breves (ej. 'Una bombilla se enciende en un instante').

\section{6. <acabar de $+V_{\text {infinitivo }}>$}

Otro de los criterios relacionados con el parámetro de la delimitación es la perífrasis terminativa < acabar de $+V_{\text {infinitivo }}>$. Así, autores como Havu (1997), De Miguel (1999) y Marín (1999), retomando el test original de Dowty (1979), afirman que solo los predicados que expresan realizaciones son compatibles con la perífrasis <acabar de $+V_{\text {infinitivo }}>$, una construcción que denota la culminación de una situación durativa. Si bien los resultados muestran inicialmente que todas las categorías aceptan en 
algún caso esta construcción, las realizaciones presentan los índices de coaparición más altos (ver Tabla 9).

Ahora bien, si nos atenemos a los resultados obtenidos para los logros, observamos que, a diferencia de los procesos y los estados, aceptan en muchas ocasiones esta construcción, en contra de la hipótesis inicial. En concreto, estos resultados se deben al hecho de que, en español, es muy frecuente el uso de la perífrasis <acabar de $+V_{\text {infinitivo }}>$ en presente para indicar los instantes inmediatamente posteriores a un evento puntual (ej. Su marido acaba de morir).

\subsection{Criterios durativos: <de repente>, <a las $X$ horas $>$, <desde hace $X$ tiempo>}

En cuanto al parámetro de la duración, desde el trabajo de Vendler (1957) se afirma que solo los verbos que expresan eventos puntuales son compatibles con modificadores adverbiales temporales de escasa duración, como <de repente> $y<a$ las $X$ horas $>$. En consecuencia, se espera que los logros muestren los índices de frecuencia más altos para estas construcciones. Los resultados confirman claramente esta hipótesis, tal como se muestra en la Tabla 10.

Sin embargo, en la bibliografía sobre los modos de acción del verbo no se cita el criterio opuesto, esto es, una construcción que solo acepten los verbos que designan eventos durativos. En este sentido, consideramos que el grado de incompatibilidad de un verbo con la construcción <desde hace $\mathbf{X}$ tiempo> podría ser un criterio adicional para identificar los logros, de ahí que también la hayamos incluido en el presente estudio. En concreto, se espera que los eventos durativos presenten índices de frecuencia más altos que los eventos puntuales (en la línea de los resultados obtenidos para <durante $X$ tiempo>). Esta predicción se confirma claramente (ver Tabla II). Los estados presentan los índices de frecuencia de coaparición más altos, mientras que los logros presentan los índices más bajos. Por lo tanto, nos hallamos ante la distribución de frecuencias inversa a la que se ha reseñado para las construcciones $<$ de repente $>\mathrm{y}<\mathrm{a}$ las $\mathrm{X}$ horas $>$.

\subsection{Tiempo verbal}

Finalmente, queda presentar los resultados relativos al tiempo verbal. Desde los trabajos de Comrie (1976) y Dahl (1985), se asume que las formas imperfectivas focalizan la estructura interna de un evento, sin expresar su principio o final, mientras que las forma perfectivas denotan un evento simple con un final bien definido. De este modo, se espera que los estados y procesos, al expresar eventos durativos no delimitados, presenten una combinación más natural con formas imperfectivas, mientras que las realizaciones y los logros, al implicar un final temporal del evento, sean más naturales con formas perfectivas. Con el fin de contrastar empíricamente esta hipótesis, se han seleccionado tres tiempos verbales: presente de indicativo (imperfectivo), pretérito imperfecto (imperfectivo) y pasado simple (perfectivo).

En términos generales, los datos muestran que

Tabla 9. <acabar de $+\mathrm{V}_{\text {infinitivo }}>$

\begin{tabular}{|c|c|c|c|}
\hline Realizaciones & Logros & Procesos & Estados \\
\hline $51,72 \%$ & $34,48 \%$ & $8,62 \%$ & $5,17 \%$ \\
\hline
\end{tabular}

Tabla 10. $<$ de repente $>$ y $<a$ las $X$ horas $>$

\begin{tabular}{|l|c|c|c|c|}
\hline & Logros & Realizaciones & Procesos & Estados \\
\hline <de repente> & $89,17 \%$ & $6,37 \%$ & $2,55 \%$ & $1,91 \%$ \\
\hline <a las X horas & $84,23 \%$ & $9,63 \%$ & $6,01 \%$ & $0,12 \%$ \\
\hline
\end{tabular}

Tabla II. <desde hace $X$ tiempo>

\begin{tabular}{|c|c|c|c|}
\hline Estados & Procesos & Realizaciones & Logros \\
\hline $65,68 \%$ & $16,42 \%$ & $16,42 \%$ & $1,48 \%$ \\
\hline
\end{tabular}


la categoría de los estados es la que presenta los índices más altos de frecuencia con el presente de indicativo y el pretérito imperfecto, así como muestra los índices más bajos con el pasado simple. De manera inversa, la categoría de los logros se sitúa en la posición más baja en cuanto al uso de tiempos imperfectivos, así como presenta los índices más altos para el pasado simple. Por lo tanto, de manera global, los resultados confirman la hipótesis de partida. La Tabla 12 presenta las frecuencias totales para las cuatro categorías analizadas.

\subsection{Síntesis del análisis intercategorial}

En esta sección se han presentado los resultados del análisis 'intercategorial'. En términos generales, se ha constatado que se establecen interacciones entre el modo de acción del verbo y los contextos gramaticales en que normalmente se usa. Asimismo, se ha mostrado que se dan patrones de distribución de frecuencia equivalentes entre construcciones que implican el mismo parámetro aspectual, así como patrones inversos para las construcciones que implican parámetros opuestos.

En concreto, respecto a las construcciones que implican sucesión de fases temporales (perífrasis progresiva y adverbios 'lentamente'/'rápidamente'), hemos observado claras diferencias de uso entre eventos durativos dinámicos y no dinámicos. En cuanto a las construcciones que implican límite temporal (<acabar de $+V_{\text {infinitivo }}>$, <en $X$ tiempo $>$ ), hemos constatado un predominio de uso de las realizaciones, aunque no siempre equivalente a los logros. A su vez, hemos comprobado que los logros muestran los índices más altos de frecuencia en los contextos que implican puntualidad (<de repente>, $<a$ las $X$ horas $>$ ), así como se diferencian del resto de categorías en construcciones que implican duración del evento (<durante $X$ tiempo>, <desde hace $X$ tiempo $>)$. En relación a las construcciones que implican habitualidad (<cada día/ semana/ mes/ año $+V_{\text {presente }}>$ ) se ha confirmado que son más frecuentes para los procesos que para los estados. En cuanto al análisis de la construcción agentiva <obligar a $+V_{\text {infinitivo }}>$, no se han encontrado diferencias entre eventos dinámicos y no dinámicos, resultados que apoyan los modelos teóricos que niegan una equiparación total entre dinamicidad y agentividad. Finalmente, se ha verificado que los eventos no delimitados, especialmente los estados, son más naturales con formas verbales imperfectivas, mientras que los eventos delimitados presentan los índices de frecuencia más altos con tiempos verbales perfectivos.

\section{Resultados del análisis intracategorial}

Hasta el momento se ha demostrado que los cuatro modos de acción del verbo, comparados entre sí, presentan diferentes patrones de uso morfosintáctico. El próximo paso es comprobar cuál es la naturaleza de la organización interna de cada categoría: itodos los miembros aceptan/ rechazan el mismo conjunto de contextos o se establecen diferencias intracategoriales? Según los resultados del presente estudio, los datos apuntan claramente a una estructuración interna gradual, con miembros más prototípicos y miembros más fronterizos. Es más, esta gradación no es arbitraria ya que se han identificado rasgos semánticos compartidos por los diferentes subconjuntos verbales. Dedicamos esta sección a presentar los resultados del análisis intracategorial.

\section{I. Estados}

Tabla I2. Tiempo verbal.

\begin{tabular}{|c|c|c|c|c|}
\hline & Estados & Procesos & Realizaciones & Logros \\
\hline Presente & $43,96 \%$ & $19,78 \%$ & $18,68 \%$ & $17,58 \%$ \\
\hline Pret. imperfecto & $44,12 \%$ & $29,41 \%$ & $|4,7| \%$ & $11,76 \%$ \\
\hline & Logros & Realizaciones & Procesos & Estados \\
\hline Pasado simple & $40,40 \%$ & $30,30 \%$ & $20,20 \%$ & $9,09 \%$ \\
\hline
\end{tabular}


En cuanto a la categoría de los estados, en primer lugar es importante apuntar que no se ha encontrado ningún caso de aparición de un verbo estativo con el adverbio 'lentamente'. Por lo tanto, es una propiedad que comparten todos los miembros de la categoría. Asimismo, hemos observado que la categoría de los estados, analizada en conjunto, muestra los índices de frecuencia más bajos en seis grandes grupos de construcciones: las que implican sucesión de fases temporales (perífrasis progresiva y adverbio 'rápidamente'); contextos delimitados (<en X tiempo>, $<$ acabar de $+V_{\text {infinitivo }}>$ ); contextos puntuales (<de repente>, <a las X horas>); habitualidad (<cada día/ mes/ semana/ año $+\mathrm{V}_{\text {presente }}>$ ); agentividad (<obligar a $+\mathrm{V}_{\text {infinitivo }}>$ ); $y$ pasado simple. Si nos centramos en estas construcciones, se pueden distinguir dos grandes patrones de uso dentro de la categoría de los estados.

Por un lado, se ha identificado un subconjunto de verbos que no acepta ningún contexto que implique progresión temporal, delimitación, puntualidad, habitualidad o agentividad, así como presenta los índices más bajos de aparición con el pasado simple ('constar', 'equivaler', 'caber', 'anteceder', 'bastar', 'consistir'). Estos verbos muestran un rasgo semántico común. En concreto, expresan propiedades físicas o constitutivas de una entidad o bien una relación, generalmente constitutiva, entre dos entidades. Otro subconjunto de predicados que presenta una distribución muy parecida a la que se acaba de exponer es el formado por las perífrasis <estar $+V_{\text {particiioio }}>$ ('estar sentado') 'enfermo'/ 'triste'/ 'preocupado'). En particular, aunque presentan índices de frecuencia intermedios para el pasado simple, rechazan el resto de contextos típicamente no estativos.

Por otro lado, encontramos un subconjunto de verbos que expresan sensaciones o pensamientos ('conocer', 'creer','gustar') que presentan los índices de frecuencia más altos -en la distribución intracategorial-en cuatro de los contextos que los estados tienden a rechazar: $<$ acabar de $+V_{\text {infinitivo }}>$, <obligar a $+V_{\text {infinitivo }}>$, pasado simple y <cada día/ semana/ mes/ año $+V_{\text {presente }}>$. La cuestión más interesante es que en estos casos la interpretación final que se deriva de la combinación 'verbo estativo + construcción dinámica', no siempre es equivalente a la que se deriva del par 'verbo dinámico + construcción dinámica'. Seguidamente veremos cuáles son estas diferencias.

En cuanto a la construcción <acabar de $+V_{\text {infinitivo }}>$, hemos observado que normalmente se usa con predicados que expresan realizaciones para focalizar la culminación del evento. No obstante, para los verbos 'creer','gustar' $y$ 'conocer' el uso de la perífrasis terminativa presenta implicaciones diferentes. En particular, para los verbos 'creer' (4a) y 'gustar' (4b) esta perífrasis se utiliza para indicar que aún no se ha llegado plenamente al estado psicológico expresado por el verbo. En cuanto al verbo 'conocer' (4c), el uso de esta perífrasis normalmente se usa para designar los instantes inmediatamente posteriores al inicio del estado. Por lo tanto, ninguna de estas interpretaciones se corresponde a la que se obtiene con las realizaciones.

(4) a. Es un sueño, no me lo acabo de creer.

b. Esa palabra no me acaba de gustar porque es inherente a la de amor.

c. No puedes mirarla como a una mujer que acabas de conocer.

En relación al pasado simple, cuando se usa con verbos dinámicos durativos -procesos y realizaciones-, la interpretación que se obtiene es la de un evento que tuvo lugar en el pasado narrativo y que no necesariamente se mantiene en el presente de la narración. No obstante, para muchos verbos que expresan estados psicológicos el pasado simple se usa para indicar el momento concreto en el que se inicia el estado; por lo tanto, una interpretación más próxima a la de un logro (5).

(5) a. Joaquín, de 25 años, conoció a la empresaria de 30 años cuando fue a Hong Kong.

b.A Virginia le gustó el médico nuevo.

c. Ella creyó que me habían asaltado.

Respecto a la construcción <cada día/ semana/ año + $\mathrm{V}_{\text {presente }}>$, cuando aparece con verbos como 'gustar' o 'creer' no adopta una interpretación habitual -como suele pasar con los verbos que expresan procesos-, sino que presenta un sentido progresivo que en muchas ocasiones se refuerza con adverbios de cantidad como 'más/ menos' (6).

(6) a. Debe ser que los años les sientan muy bien porque cada día gustan más al público.

b. Me inclino a creer, cada día con más consistencia, que también fui engañado.

Finalmente, en cuanto a la construcción <obligar $a+V_{\text {infinitivo }}>$, aunque no implica interpretaciones esencialmente diferentes a las que se obtienen con verbos dinámicos, creemos importante presentar algunos ejemplos, en tanto que los verbos 'conocer' 
y 'creer' son de los pocos verbos de estado de la muestra que aceptan esta construcción agentiva (7).

(7) a. Los profesores estan obligados a conocer el catalán y el castellano.

b. Se obligó a creer en esas leyendas.

Hasta el momento, por lo tanto, hemos identificado dos grandes patrones de uso intracategorial de los estados. Ahora bien, en este punto hay que tener en cuenta que los estados no solo se pueden definir a partir de los contextos que más rechazan, sino también a partir de los contextos que más aceptan. En concreto, según datos del análisis intercategorial, la categoría de los estados muestra los índices de frecuencia más altos en dos construcciones: presente de indicativo $y<$ desde hace $X$ tiempo $>$. Por lo tanto, es preciso comprobar si la distribución intracategorial observada hasta el momento se mantiene al analizar estos dos últimos contextos.

En cuanto al uso del presente de indicativo, los verbos que expresan estados típicamente permanentes ('consistir', 'equivaler', 'caber', 'bastar', 'anteceder', 'constar') presentan los porcentajes de frecuencia más altos, seguidos de los predicados que expresan estados transitorios ('estar sentado'/ 'enfermo'/ 'triste'/ 'preocupado'). En las posiciones más bajas encontramos verbos que expresan pensamientos o emociones ('creer', 'conocer', 'saber', 'gustar', 'odiar'). Respecto a la construcción <desde hace $X$ tiempo>, los predicados que más la aceptan son las perífrasis <estar $+V_{\text {participio }}>$ y algunos verbos psicológicos ('conocer', 'creer', 'merecer', 'desear'), como se muestra en (8).

(8) a. Está enfermo desde hace años.

b.Abordará la situación económica en un debate deseado por Pujol desde hace ya meses.

c. Lo conozco desde hace 25 o 30 años.

Contrariamente, no encontramos ejemplos de verbos que expresen estados permanentes con esta construcción. Por lo tanto, en principio se podría pensar que estos resultados se desvían de la tendencia observada hasta el momento. No obstante, consideramos que estos datos en realidad confirman el hecho de que los verbos que expresan estados permanentes son los más prototípicos de la categoría: al ser inherentemente durativos, se conciben de manera holística, motivo por el cual generalmente no aparecen con construcciones que acotan la extensión del evento, como es el caso de la construcción < desde hace $\mathrm{X}$ tiempo>.

En definitiva, si bien la categoría de los estados, analizada en conjunto, muestra patrones de uso significativamente diferentes de las categorías aspectuales dinámicas, observamos que incluye miembros más prototípicos que otros, datos que apuntan a una organización interna gradual de la categoría. En particular, se pueden distinguir como mínimo tres grandes subtipos aspectuales dentro de la categoría de los estados que presentan diferentes grados de prototipicidad:

a) Verbos que expresan propiedades físicas o constitutivas de una entidad (generalmente un objeto). Estos verbos designan situaciones inherentemente estables y durativas, razón por la cual no admiten contextos dinámicos, presentan los índices de frecuencia más altos para el presente de indicativo, así como generalmente no coaparecen con construcciones que indican explícitamente el grado de duración de la situación. En consecuencia, son miembros prototípicos de la categoría de los estados.

b) Predicados que expresan una propiedad física - emocional de una entidad (generalmente una persona) que solo se mantiene para un periodo temporal determinado. Estos predicados, si bien rechazan la mayoría de contextos dinámicos, presentan índices de frecuencia intermedios para el pasado simple y permiten acotar el periodo temporal en el cual se mantiene la situación. Por lo tanto, se situarían en una posición intermedia dentro de la categoría de los estados.

c) Verbos de pensamiento o emoción. Son verbos que, dentro de la categoría de los estados, presentan los índices de frecuencia de coaparición más altos para las construcciones dinámicas, muestran los índices más bajos para el presente de indicativo, así como permiten delimitar el periodo temporal de la situación que denotan. Por lo tanto, son los verbos más fronterizos de la categoría.

\subsection{Procesos}

A partir del análisis intercategorial, hemos comprobado que la categoría aspectual de los procesos es la que presenta los índices de frecuencia más altos con cuatro construcciones gramaticales: perífrasis progresiva, <durante $X$ tiempo $>$, adverbio 'lentamente' y <cada día/ semana/ año $+V_{\text {presente }}>$. 
Los procesos no presentan los índices de frecuencia más bajos en ninguna de las construcciones analizadas. Por lo tanto, el análisis se centra únicamente en los contextos de mayor aceptación. De entrada, no se han encontrado diferentes patrones de uso intracategorial con la construcción habitual. Por lo tanto, estos datos sugieren que la habitualidad no es una propiedad determinante de la organización interna de la categoría de los procesos. Contrariamente, si nos basamos en el resto de construcciones, se establecen diferentes patrones de uso intracategorial que nos permiten distinguir tres grandes tipos de verbos que expresan procesos.

En primer lugar, hemos identificado un subconjunto de ítems léxicos que presentan las frecuencias más altas de aparición con la perífrasis progresiva y la construcción <durante $X$ tiempo>. No obstante, presentan porcentajes de frecuencia muy bajos para el adverbio 'lentamente'. El conjunto de verbos es el siguiente: 'pensar', 'investigar', 'estudiar', 'esperar', 'buscar', 'mirar' y 'trabajar'. Observamos que todos son verbos que designan eventos que requieren algún tipo de actividad agentiva y que no necesariamente implican un movimiento o desplazamiento físico de una entidad. En (9) se muestran algunos ejemplos de este subconjunto verbal.

(9) a. Estoy pensando continuamente en ella.

b. La policía está investigando el asesinato de dos vagabundos.

c. Los alumnos deberán estudiar estas materias durante II cursos.

d. Miles de personas les esperaron durante horas en la plaza del Pilar.

En este punto es importante aclarar que el adverbio 'lentamente', si bien es uno de los criterios más utilizados para detectar los verbos de proceso, requiere cierta precaución. Este adverbio no solo se refiere al progreso de un evento en el tiempo sino también al ritmo o velocidad de ese progreso. En consecuencia, los verbos de proceso que no implican un ritmo cambiante rechazan este adverbio. En este sentido, Van Valin (2005), por ejemplo, prefiere el uso de otros adverbios como 'activamente' o 'enérgicamente' para detectar la prototipicidad de los verbos de proceso. Si nos basamos en este criterio alternativo y lo aplicamos a todos los verbos de proceso de la muestra, comprobamos que el adverbio 'activamente' solo aparece con este primer subgrupo de procesos: verbos agentivos que no implican movimiento o desplazamiento físico. En (I0) se muestran algunos ejemplos.

(I0) a. Continúan trabajando activamente en la campaña electoral.

b. El rey de la droga era investigado activamente por la DEA y el FBI.

c. [...] cuyo objetivo es obligar a los parados a "salir del subsidio" para buscar activamente un empleo.

d.Deberíamos estudiar activamente los aspectos positivos de la iniciativa.

En segundo lugar, hemos detectado un subconjunto de verbos que, si bien rechazan el adverbio 'activamente', así como presentan posiciones intermedias para la perífrasis progresiva y la construcción <durante $X$ tiempo>, se sitúan entre las 10 primeras posiciones de frecuencia de coaparición con el adverbio 'lentamente'. El listado de verbos es el siguiente: 'caminar', 'pasear', 'acariciar', 'empujar' y 'ascender'. Notamos que en este caso también son verbos que presentan un rasgo semántico común: expresan procesos agentivos que implican algún tipo de desplazamiento físico de una entidad. Podemos ver algunos ejemplos en (I I).

(I I) a.Venías a mi lado, paseando lentamente entre aquellos eucaliptos.

b. Garfio está acariciando lentamente, en silencio, la mano izquierda de Peter Pan.

c. Empujó la aguja lentamente hasta que salió por otro lugar.

Finalmente, encontramos un tercer subconjunto verbal dentro de la categoría de los procesos que, si bien presenta índices altos de frecuencia con la forma progresiva, no aparece en ningún caso con el adverbio 'lentamente' ni con el adverbio 'activamente', así como muestra los índices más bajos de aparición con <durante $X$ tiempo>. Por lo tanto, sería el subconjunto menos prototípico de la categoría de los procesos. El listado de verbos es el siguiente: 'temblar', 'gritar', 'roncar', 'llorar', 'reir' $y$ 'soñar'. En este caso, también comparten un rasgo semántico común: son ítems léxicos que expresan algún tipo de proceso físico que experimenta un individuo, generalmente de forma no agentiva.

En definitiva, tal como se ha constatado para el análisis intracategorial de los estados, los datos muestran que la organización interna de los 
procesos también se estructura de manera gradual, con miembros más prototípicos que otros. En particular, hemos comprobado que se establecen tres grandes subtipos intracategoriales: procesos agentivos que no implican desplazamiento físico, procesos agentivos que requieren desplazamiento físico y procesos con un sujeto experimentador.

\subsection{Realizaciones}

En cuanto a las realizaciones, según datos del análisis intercategorial, es la categoría que muestra los índices de frecuencia más altos en cinco construcciones: <en $\mathrm{X}$ tiempo $>$, <acabar de $+\mathrm{V}_{\text {infinitivo }}>$, <obligar a $+\mathrm{V}_{\text {infinitivo }}>$, 'lentamente' y 'rápidamente'.

En primer lugar, es importante subrayar que se ha detectado un subconjunto de verbos que, dentro de la categoría de las realizaciones, se sitúan entre las 10 primeras posiciones de frecuencia en todos los contextos que se acaban de exponer.Por lo tanto, se comportan claramente como verbos prototípicos de la categoría ('construir', 'destruir', 'aprender', 'recorrer', 'instalar(se)', 'hundir(se)', 'convertir(se)', 'recuperar(se)'). Notamos que todos son verbos transitivos o que presentan una alternancia entre una variante transitiva y una pronominal. Además, son predicados que generalmente implican un tema afectado por la acción. En (I2) se presentan algunos ejemplos.

(I2) a. Una popular rúa recorrió en dos horas todo el perímetro de la animada Rambla.

b. Está acabando de construir un rancho con $\mathbf{4 0}$ caballos.

c. Están obligados a instalar este sistema de seguridad.

d. Se recupera lentamente, según fuentes del hospital norteamericano.

e. Ena, con tesón típicamente inglés, aprendió el castellano rápidamente.

En segundo lugar, si nos atenemos a los ítems léxicos que presentan los índices de frecuencia más bajos en estos contextos, encontramos el siguiente subconjunto de verbos: 'decidir', 'solucionar', 'aparcar', 'fabricar', 'fundir' y 'solidificar'. En concreto, por un lado tenemos verbos que, si bien implican un proceso previo, focalizan la culminación de un evento ('decidir', 'solucionar', 'aparcar') y, por otro lado, verbos que, si bien expresan una situación que implica un cambio de estado, focalizan el proceso gradual que lleva al cambio ('fabricar', 'fundir', 'solidificar'). Por lo tanto, los primeros serían más próximos a los logros y los segundos más próximos a los procesos.

\subsection{Logros}

Finalmente, queda presentar los resultados obtenidos para la categoría de los logros. Como se ha observado en el análisis intercategorial, la categoría de los logros presenta los porcentajes más altos de aparición con contextos que implican puntualidad del evento (<de repente>, <a las X horas>) y con el pasado simple. De entrada, si nos basamos en estos contextos, no encontramos diferentes patrones o subtipos intracategoriales, datos que apuntarían a una distribución homogénea de la categoría. Ahora bien, en este punto hay que tener presente que los verbos que expresan logros no solo son los que más aceptan los contextos puntuales, sino también los que más rechazan los contextos durativos (<durante $X$ tiempo>, <desde hace $X$ tiempo>, perífrasis progresiva y presente de indicativo). Es precisamente a partir del análisis de estas últimas construcciones que también observamos una organización gradual de la categoría de los logros.

En concreto, hemos identificado un subconjunto de verbos ('atrapar', 'capturar', 'detectar', 'heredar', 'adivinar', 'enterarse') que se sitúan entre las 10 posiciones más bajas de frecuencia para todos los contextos durativos. Estos datos sugieren que son verbos prototípicos de la categoría. Por otro lado, encontramos un subconjunto de verbos que, aunque son de los más citados en la bibliografía como ejemplos de logros, se comportan como verbos fronterizos de la categoría, según datos del estudio de corpus ('perder', 'ganar', 'abrir', 'cerrar'), ya que presentan los índices de frecuencia más altos para la perífrasis progresiva, y son de los pocos ítems de la categoría que aceptan la construcción <durante $X$ tiempo>. Podemos ver algunos ejemplos en (I3).

(I3) a.Yo ya había puesto la mesa hacía rato y estaba abriendo una botella de vino.

b. La izquierda estaba ganando por un 55 por ciento de los votos emitidos.

c. [...] cerraron las instalaciones durante una semana en señal de duelo.

d. [...] perdió la titularidad durante varios meses.

Por lo tanto, notamos que son verbos que permiten focalizar el estadio anterior (proceso) o posterior (estado) del evento expresado. Es decir, cuando se usan con la forma progresiva son más próximos a las realizaciones, y cuando aparecen con la construcción 
<durante $X$ tiempo> se comportan como estados, expresando la duración del estado resultante de un logro.

\subsection{Síntesis del análisis intracategorial}

En definitiva, a partir del análisis intracategorial de los datos, se ha validado empíricamente otra de las hipótesis de partida básicas del presente trabajo: la organización interna gradual de las categorías aspectuales. En particular, se ha mostrado que en todos los casos se pueden identificar miembros más prototípicos y miembros más flexibles de cada categoría, una gradación que no es arbitraria sino que se configura a partir de rasgos semánticos compartidos por los diferentes subconjuntos verbales. En total, se han identificado II subtipos aspectuales que se recogen en la Tabla I3 a modo de síntesis.

Además, es importante destacar que los posibles cambios de los verbos más flexibles -o fronterizostampoco se dan de forma arbitraria; contrariamente, siguen una sistematicidad conceptual: la gradación se efectúa entre categorías contiguas que comparten algún parámetro aspectual. En la Figura I se representa de manera gráfica la relación que se establece entre los cuatro grandes modos de acción del verbo, así como entre los diferentes subtipos aspectuales, según los resultados del presente estudio de corpus.

Tabla 13. Síntesis de subtipos aspectuales.

\begin{tabular}{|c|c|c|}
\hline CATEGORÍAS & SUBTIPOS & EJEMPLOS \\
\hline \multirow[t]{3}{*}{ Estados } & Permanentes & consistir, equivaler, caber \\
\hline & Transitorios & estar enfermo, triste \\
\hline & $\begin{array}{c}\text { Psicológicos } \\
{[+ \text { pasado simple, acabar de }]=\text { logro }} \\
{[+ \text { cada día más } / \text { menos }]=\text { proceso }}\end{array}$ & conocer, gustar, creer \\
\hline \multirow[t]{3}{*}{ Procesos } & Sujeto experimentador & temblar, llorar, roncar \\
\hline & Agentivos sin desplazamiento físico & pensar, esperar, mirar \\
\hline & Agentivos con desplazamiento físico & caminar, pasear, correr \\
\hline \multirow[t]{3}{*}{ Realizaciones } & Foco proceso & fabricar, solidificar \\
\hline & Prototípicas: transitivas y tema afectado & construir, destruir \\
\hline & Foco culminación & decidir, aparcar \\
\hline \multirow[t]{2}{*}{ Logros } & Prototípicos: puntuales & atrapar, detectar, enterarse \\
\hline & $\begin{array}{c}\text { Fronterizos: } \\
{[+ \text { progresivo }]=\text { realización }} \\
{[+ \text { durante } X \text { tiempo }]=\text { estado }}\end{array}$ & perder, cerrar, abrir \\
\hline
\end{tabular}




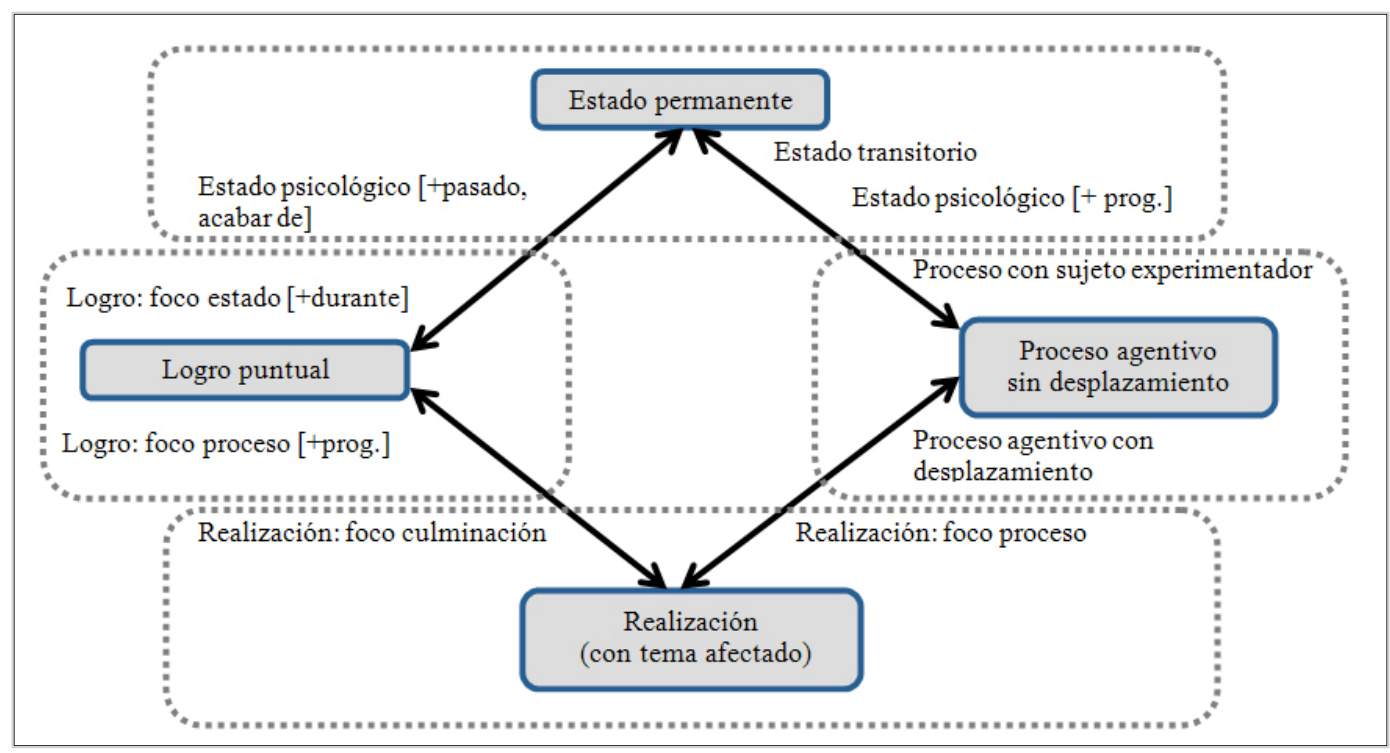

Figura I. Relación entre modos de acción del verbo.

\section{CONCLUSIONES}

En este artículo se ha presentado un estudio empírico sobre los cuatro grandes modos de acción del verbo basado en datos del español extraídos de corpus. En particular, se han analizado un total de I.680 contextos a partir del cruce de 120 verbos y 14 construcciones. En términos generales, los resultados muestran una clara interacción entre la categoría aspectual de un verbo y su frecuencia de aparición con determinadas construcciones gramaticales. De este modo, se ha validado empíricamente el hecho de que la pieza léxica verbal presenta propiedades semánticas relacionadas con el aspecto que imponen restricciones morfosintácticas. Además, los resultados muestran distribuciones de uso intercategoriales equivalentes entre construcciones que implican un mismo parámetro aspectual, así como distribuciones inversas para las construcciones que implican parámetros opuestos (ej. construcciones durativas vs. puntuales). En última instancia, por lo tanto, se confirma la realidad del aspecto léxico.

No obstante, se ha demostrado que la organización interna de dichas categorías no es homogénea, como se postula desde las aproximaciones lexicalistas tradicionales; contrariamente, presentan una estructura interna gradual,con miembros prototípicos que solo admiten construcciones coherentes con el tipo de evento básico que expresan, y miembros más flexibles que admiten cambios de interpretación aspectual. En otras palabras, dentro de una categoría aspectual se establecen diferentes pesos léxicos en la interacción entre semántica léxica y contexto morfosintáctico. Es más, se ha demostrado que esta gradación interna no es arbitraria:por un lado, se han identificado rasgos semánticos compartidos por los diferentes subgrupos verbales intracategoriales (ej. permanencia/transitoriedad, agentividad,movimiento, etc.); $y$, por otro lado, se ha comprobado que el cambio de interpretación aspectual de los verbos más flexibles sigue una sistematicidad conceptual, ya que el cambio se efectúa entre categorías contiguas que comparten algún parámetro aspectual.

En definitiva, este estudio presenta una nueva propuesta de clasificación de los modos de acción del verbo que permite cubrir dos de las carencias básicas de los trabajos realizados hasta el momento, puesto que se basa en una muestra amplia y variada de predicados $y$, de manera más relevante, ofrece una clasificación probada empíricamente a partir de contextos de uso reales extraídos de corpus. Finalmente, y de manera relacionada, se aporta una nueva metodología para la identificación de rasgos semánticos que se puede hacer extensiva al estudio de otros aspectos del significado. 


\section{REFERENCIAS BIBLIOGRÁFICAS}

Aarts, B. (2004). Modelling linguistic gradience. Studies in Language, 28(I), I -49.

Bertinetto, P. (1987). II dominio tempo-aspettuale: Demarcazioni, intersezioni, contrasti. Torino: Rosenberg \& Sellier.

Binnick, R. (|99|). Time and the verb. A guide to tense and aspect. Oxford: Oxford University Press.

Borer, H. (1994). The projection of arguments. En E. Benedicto \& J. Runner (Eds.), Functional projections (pp. I79-208).Amherst: GLSA.

Comrie, B. (1976). Aspect: An introduction to the study of verbal aspect and related problems. Cambridge: Cambridge University Press.

Croft, W. (2008). Aspectual and causal structure in event representations. En V. Gathercole (Ed.), Routes to language development. Studies in honor of Melissa Bowerman (pp. I39- I66). Mahwah, NJ: Erlbaum.

Dahl, O. (1985). Tense and aspect systems. Oxford: Blackwell.

De Miguel, E. (1999). El aspecto léxico. En I. Bosque \& V. Demonte (Eds.), Gramática descriptiva de la lengua española (pp. 2977-3060). Madrid: Espasa Calpe.

De Miguel, E. (2004). Qué significan aspectualmente algunos verbos y qué pueden llegar a significar. Estudios de Lingüistica, 18, 167-206.

Demonte, V. (2006). Qué es sintáctico y qué es léxico en la interficie entre sintaxis y léxicosemántica: Hipótesis y conjeturas. Signo y Seña, I5, $17-42$.

Dowty, D. (1979). Word meaning and Montague grammar:The semantics of verbs and times in generative semantics and in Montague.s PTQ. Dordrecht: Reidel.

Gennari, S. \& Poeppel, D. (2003). Processing correlates of lexical semantic complexity. Cognition, 89, 27-4I.

Havu, J. (1997). La constitución nominal del sintagma verbal en el español moderno. Helsinki: Academia Scientiarum Fennica.

Jackendoff, R. (1990). Semantic structures. Cambridge: MIT Press.

Jackendoff, R. (|99|). Parts and boundaries. Cognition, 4I, 9-45.

Jackendoff, R. (2002). Foundations of language. Oxford: Oxford University Press.

Lakoff, G. (1970). Irregularity in syntax. Nueva York: Rinehart and Winston.

Levin, B. \& Rappaport Hovav, M. (I995). Unaccusativity: At the lexical syntax-semantics interface. Cambridge, MA: MIT Press.

Marín, R. (2000). El componente aspectual de la predicación. Tesis doctoral, Universitat Autónoma de Barcelona, Barcelona, España.

Mittwoch, A. (I99I). In defence ofVendler's achievements. Belgian Journal of Linguistics, 6, 7 I-85.

Mourelatos, A. (1978). Events, processes and states. Linguistics and Philosophy, 2, 4I5-34.

Pustejovsky, J. (I99I). The syntax of event structure. Cognition, 4I, 47-8I.

Ritter, E. \& Rosen, S. (1998). Delimiting events in syntax. En M. Butt \&W. Geuder (Eds.), The projection of arguments (Pp. I35- I64). Stanford: CSLI Publications. 
Smith, C. (199I). The parameter of aspect. Dordrecht: Kluwer.

Van Valin, R. (2005). Exploring the syntax-semantics interface. Cambridge: Cambridge University Press.

Vendler, Z. (1957). Verbs and times. The Philosophical Review, 66(2), I43-160.

Verkuyl, H. (1989). Aspectual classes and aspectual composition. Linguistics and Philosophy, I2, 39-64. 


\section{ANEXO}

\section{Lista de verbos considerados en el análisis de corpus}

\begin{tabular}{|c|c|c|c|}
\hline ESTADOS & PROCESOS & REALIZACIONES & LOGROS \\
\hline abundar & acariciar & acceder & abrir \\
\hline amar & ascender & acudir & acertar \\
\hline anteceder & bailar & aparcar & apagar \\
\hline bastar & buscar & aprender & atrapar \\
\hline caber & caminar & beber & callar \\
\hline carecer & correr & comer & capturar \\
\hline conocer & empujar & concretar & caer \\
\hline consistir & escuchar & congelar & cerrar \\
\hline constar & esperar & construir & chocar \\
\hline creer & estudiar & convencer & descubrir \\
\hline depender & explorar & convertir & desmayarse \\
\hline desear & fumar & decidir & despertar \\
\hline equivaler & gritar & destruir & detectar \\
\hline estar enfermo & investigar & dibujar & disparar \\
\hline estar triste & jugar & elaborar & encender \\
\hline estar preocupado & Ilorar & escribir & enterarse \\
\hline estar enfadado & mecer & fabricar & ganar \\
\hline estar sentado & mirar & fundir & heredar \\
\hline existir & nadar & hundirse & iniciar \\
\hline gustar & observar & instalar & localizar \\
\hline merecer & pasear & leer & lograr \\
\hline necesitar & pensar & pintar & morir \\
\hline odiar & perseguir & recorrer & nacer \\
\hline pertenecer & reír & recuperarse & obtener \\
\hline poseer & roncar & reparar & perder \\
\hline preferir & sonar & resolver & recibir \\
\hline permanecer & soñar & resumir & reconocer \\
\hline residir & temblar & solidificar & surgir \\
\hline sobrar & trabajar & solucionar & topar \\
\hline temer & viajar & volver & tropezar \\
\hline
\end{tabular}

* Esta investigación se ha llevado a cabo gracias al proyecto KNOW2 (TIN2009-|47I5-C04) del Ministerio Español de Ciencia e Innovación. 